\title{
The Study on Changes of NKT Cells Levels in Peripheral Blood of Children with Asthma
}

\author{
Huilan Zhu ${ }^{1, a}$, Xinxin Xin ${ }^{2,}$, , Hongyan Zhang ${ }^{1}$ \\ ${ }^{1}$ Department of Pediatrics, The first people's Hospital of Ji'nan, Ji'nan,250011,China \\ ${ }^{2}$ Department of Surgery, The fifth people's Hospital of Ji'nan, Ji'nan,250022,China \\ azhu666@126.com
}

*Corresponding author xinxin@126.com

Keywords: Asthma; Nature killer cell; T cell

\begin{abstract}
Objective: To study the changes of natural killer T cells (NKT cells)in peripheral blood and the ability of NKT cells to proliferate in response. Methods: 80 bronchial asthma children and 40 healthy children were included. NKT cells and $\mathrm{T}$ cells were analyzed by flow cytometry instrument. The levels of IL-4, IFN- $\gamma$, IL-10 were tested in different period of NKT cell culture. Results: There were 46 cases were acute, 34 cases were remission. Between the three groups, CD4+, $\mathrm{CD} 8+, \mathrm{CD} 4+/ \mathrm{CD} 8+$ and NKT cells percentage comparison difference were statistically significant $(\mathrm{P}<0.05)$. CD4 +, CD4 + / CD8 + percentage in acute episodes were higher than the remission group and normal group, but CD $8+$, NKT cells percentage were lower than the remission group and normal group. The difference was statistically significant $(\mathrm{P}<0.05)$. There were no statistically significant difference he remission group between remission group and normal group $(\mathrm{P}>0.05)$. With the extension of incubation time, IL -4 levels in three groups of children were significantly increased, and acute attack group were higher than that in remission group and normal group. The difference was statistically significant $(\mathrm{P}<0.05)$. Serums IL - 10 in cultivating $48 \mathrm{~h}$ raised highest, cultivate $72 \mathrm{~h}$ declined, and acute attack group were higher than that in remission group and normal group. The difference was statistically significant $(\mathrm{P}<0.05)$. Conclusion: The pathogenesis of asthma may be related to the ratio and dysfunction of NKT cells.
\end{abstract}

\section{Introduction}

The bronchial asthma is by a variety of cells, including, the structure of the airway inflammatory cells and cellular components involved in chronic inflammatory airway disease, is one of the most common chronic respiratory disease polio. Clinical asthma pathogenesis pattern is more because of the simple fruit pathogenic mode, biological genetic factors play a role in physiological primordial, is an important pathogenic internal cause. Findings, natural killer T cells (NKT cells) stimulation can produce immunity factor, leading to immune function imbalance, it may be involved in the occurrence and development process of asthma. In this context, this study is to changes in different period of children with asthma NKT cells for research; provide a reference for clinical research.

\section{Materials and Methods}

\section{Materials}

We continuously collected 80 cases of clinic of our hospital diagnosed as bronchial asthma children, male 43 patients, 37 cases of women, age (3-13), mean age $8.2 \pm 2.1$ years of age, children have different degree of wheezing, coughing, shortness of breath, chest tightness and other symptoms of acute phase, or the original symptoms increase sharply, the diagnosis of paediatrics' branch of Chinese medical association respiratory group of the children bronchial asthma diagnosis and control guide. At the same time choose 40 cases of healthy children, 40 cases of healthy children as the research object, male 22 cases, 18 cases, women aged (3-13), mean age 8.1 2.1 years old. Exclusion criteria: lung infection, associated with other lung diseases, malignant tumour, 
blood coagulation dysfunction, connective tissue diseases, etc. All the families of children with sign the informed consent.

\section{Methods}

We perfect the relevant inspection, test, according to the cases given oxygen, given antibiotic resistance to infection, phlegm cough, correct electrolyte disorder and corresponding support therapy measures such as to maintain acid-base balance.

Check all of them to return sputum, told the child to gargle with physiological saline, and then turn back to help children with deep eliminate sputum, cough up phlegm liquid after leave sterile bottles of BOC pathogen examination. Using flow cytometry instrument for all sputum samples of T cell subsets CD3 +, CD4 + and CD3 + CD8 +, CD4 + CD45RA +, CD8 + CD45RA + is analyzed. Extraction was hollow in the middle of venous blood $2 \mathrm{ml}, 3000 \mathrm{r} / \mathrm{min}$, the centrifugal $5 \mathrm{~min}$, stored in $-80{ }^{\circ} \mathrm{C}$ refrigerator waiting for inspection. By immune fluorescence labelling and flow cytometry analysis of peripheral blood of NKT cells. The NKT cells cultivation using enzyme-linked immunosorbent method detection (T1) 24 h, 48 h (T2), 72 h (T3) serum IL - 4, IFN gamma, IL - 10 levels.

\section{Statistical processing}

With mathematical statistics software SPSS19.0 for data collection and statistical analysis, and chi-square test, $\mathrm{P}<0.05$, significant difference has statistical significance.

\section{Results}

\section{The data analysis in general}

80 patients diagnosed with asthma, in children with acute onset of 46 cases, 25 cases of male,

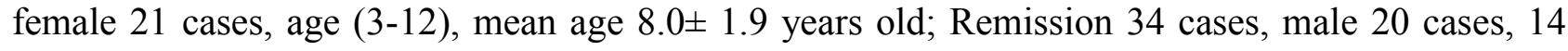
cases of women, age (3-13), mean age $8.2 \pm 2.3$ years old. Compared three groups of children with general data there was no statistically significant difference $(\mathrm{P}>0.05)$, and can be used as a clinical comparative object, (The following table 1).

Table 1: The data analysis in general

\begin{tabular}{ccccc}
\hline \multirow{2}{*}{ Group } & \multicolumn{4}{c}{ Gender } \\
& Male & Female & Age \\
\hline $\begin{array}{c}\text { Acute group } \\
\text { The } \\
\begin{array}{c}\text { remission } \\
\text { group }\end{array}\end{array}$ & 46 & 25 & 21 & $8.0 \pm 1.9$ \\
$\begin{array}{c}\text { The normal } \\
\text { group } \\
\chi^{2} \text { Value }\end{array}$ & 40 & 25 & 14 & $8.2 \pm 2.3$ \\
P Value & & 1.021 & $8.1 \pm 2.1$ \\
\hline
\end{tabular}

\section{Three groups of children with peripheral blood $T$ cells and NKT cells}

Three groups of children with peripheral blood $\mathrm{T}$ cells and NKT cells comparison results showed that among three groups of CD4 +, CD8 +, CD4 + / CD8 + and NKT cells percentage comparison difference was statistically significant $(\mathrm{P}<0.05)$, acute episodes of CD4 +, CD4 + / CD8 + percentage is higher than the remission group and normal group, CD8 +, NKT cells percentage is lower than the remission group and normal group, the difference was statistically significant $(\mathrm{P}<$ $0.05)$, the remission group there was no statistically significant difference compared with normal group ( $>$ > 0.05); CD3 + and CD3 + CD56 + and CD3 - the percentage of CD56 + comparison difference is not obvious ( $\mathrm{P}>0.05)$, (The following table 2$)$. 
Table 2: Three groups of children with peripheral blood T cells and NKT cells

\begin{tabular}{cccccc}
\hline Group & $\begin{array}{c}\text { Acute group } \\
(\mathrm{n}=46)\end{array}$ & $\begin{array}{c}\text { The remission } \\
\text { group }(\mathrm{n}=34)\end{array}$ & $\begin{array}{c}\text { The normal } \\
\text { group }(\mathrm{n}=40)\end{array}$ & $\begin{array}{c}\mathrm{t} \\
\text { Value }\end{array}$ & $\begin{array}{c}\mathrm{P} \\
\text { Value }\end{array}$ \\
\hline $\mathrm{CD} 3+(\%)$ & $69.26 \pm 7.22$ & $69.16 \pm 8.47$ & $68.83 \pm 7.23$ & 0.655 & 0.326 \\
$\mathrm{CD} 4+(\%)$ & $43.33 \pm 6.54$ & $33.35 \pm 5.56$ & $32.74 \pm 5.54$ & 3.986 & 0.034 \\
$\mathrm{CD} 8+(\%)$ & $21.64 \pm 9.23$ & $29.94 \pm 9.45$ & $29.17 \pm 3.73$ & 4.027 & 0.029 \\
$\mathrm{CD} 3+\mathrm{CD} 56+(\%)$ & $3.03 \pm 0.72$ & $3.03 \pm 0.81$ & $2.97 \pm 0.73$ & 0.988 & 0.134 \\
$\mathrm{CD} 3-\mathrm{CD} 56+(\%)$ & $9.64 \pm 1.23$ & $10.94 \pm 1.44$ & $10.74 \pm 1.23$ & 0.729 & 0.212 \\
$\mathrm{CD} 4+/ \mathrm{CD} 8+(\%)$ & $2.13 \pm 0.54$ & $1.33 \pm 0.33$ & $1.24 \pm 0.24$ & 1.489 & 0.069 \\
NKT Cell $(\%)$ & $0.040 \pm 0.015$ & $0.078 \pm 0.019$ & $0.141 \pm 0.043$ & 3.117 & 0.045 \\
\hline
\end{tabular}

\section{Three group of NKT cells in children with amplification different time's cytokine level analysis}

Three groups of children with NKT cells amplify different times cytokine levels analysis results showed that with the extension of incubation time, three groups of children were significantly increased serum IL - 4, level and different periods of acute attack group were higher than that of remission group and normal group, the difference was statistically significant $(\mathrm{P}<0.05)$; Serum IL 10 in cultivating $48 \mathrm{~h}$ rise highest, cultivate $72 \mathrm{~h}$ decline, levels and different periods of acute attack group were higher than that of remission group and normal group, the difference was statistically significant $(\mathrm{P}<0.05)$; Three groups of children with serum levels of IFN - gamma comparison difference is not obvious $(\mathrm{P}>0.05)$, (see table 3$)$.

Table 3: Three group of NKT cells in children with amplification different time' s cytokine level analysis

\begin{tabular}{ccccc}
\hline \multicolumn{1}{c}{ Group } & Indicators & T1 & T2 & T3 \\
\hline \multirow{2}{*}{$\begin{array}{c}\text { Acute group } \\
(\mathrm{n}=46)\end{array}$} & IL-4(ng/L) & $30.83 \pm 4.23$ & $112.91 \pm 9.43$ & $214.08 \pm 12.23$ \\
& IFN- $\gamma(\mathrm{ng} / \mathrm{L})$ & $117.74 \pm 18.54$ & $421.57 \pm 28.13$ & $228.45 \pm 18.44$ \\
& IL-10(ng/L) & $11.17 \pm 2.73$ & $24.38 \pm 3.13$ & $12.81 \pm 1.31$ \\
$\begin{array}{c}\text { The remission } \\
\text { group }\end{array}$ & IL-4(ng/L) & $9.65 \pm 2.73$ & $42.76 \pm 8.13$ & $73.43 \pm 8.31$ \\
$\quad$ IFN-34) $\gamma(\mathrm{ng} / \mathrm{L})$ & $139.74 \pm 19.73$ & $413.82 \pm 18.32$ & $231.62 \pm 18.55$ \\
& IL-10(ng/L) & $36.45 \pm 3.24$ & $95.45 \pm 5.44$ & $59.38 \pm 5.43$ \\
$\begin{array}{l}\text { The normal } \\
\text { group } \\
(\mathrm{n}=40)\end{array}$ & IL-4(ng/L) & $9.17 \pm 1.73$ & $41.38 \pm 8.13$ & $72.82 \pm 9.31$ \\
& IFN- $\gamma(\mathrm{ng} / \mathrm{L})$ & $140.74 \pm 19.73$ & $414.82 \pm 18.45$ & $222.62 \pm 18.55$ \\
\hline
\end{tabular}

\section{Discussion}

Bronchial asthma is by a variety of cells in particular, mast cells and $\mathrm{T}$ lymphocytes in chronic airway allergic inflammation, clinical mainly characterized by recurrent episodes of wheezing, difficulty breathing, chest tightness, and coughing, much in the night and early in the morning. A lot of research is in nearly 10 years although asthma, the progress is very big, and its incidence is still on the rise. According to a 2002 Chinese children asthma epidemiology investigation shows, prevalence of 0.5 to $3.3 \%$ two years, rose by $64.6 \%$ over the past ten years. Estimates about the 
country are more than 2000 asthma patients. Developed countries such as United States, Britain, the prevalence rate is as high as $20-30 \%$, mortality is also on the rise, so the asthma has become a threat to human especially the serious public health problem of children's health, is causing great concern in many countries around the world. According to the current understanding of the pathogenesis of asthma, treatment focuses on application of anti-inflammatory, beta 2 agonists, the drug can significantly improve the chronic airway inflammation, relieve the airway, but systemic side effects, especially long inhaled treatment, high technical requirements, children is not easy, so the clinical application is limited. Traditional Chinese medicine to treat asthma has accumulated rich experience, based on evidence-based medicine in the treatment, from the overall point of view combined with individual factors, curative effect affirmation, small side effects, it not only take temporary solution and effect a permanent cure, pathogenesis, which can obviously reduce the recurrence rate.

Childhood is the sensitive stage of asthma attacks, as a kind of common chronic respiratory allergic disease, its worldwide in recent years gradually increasing trend. Clinically, when acute bronchial asthma in children with irritability, shrug, difficult to breath more significantly, pale face, nose flap, oral and nails purple, whole body cold sweat, auxiliary well contraction, in a chest tightness, shortness of breath, even when speaking words not continuous. Studies have confirmed that, the occurrence and development of $\mathrm{T}$ cells in asthma play a major role in the process, but the Th2 immune mechanism can only explain allergen Th2 exist a variety of characteristics of asthma, but there is no specific Th2 to participate in the deterioration of the situation can not explain. NKT cells are a group of cell surfaces both TCR T cell receptors, and there was a special T cell subsets of NK cell receptors.

This study analysis of three groups of children with peripheral blood T cells and NKT cells found that acute group of CD4 +, CD4 + / CD8 + percentage is higher than the remission group and normal group, $\mathrm{CD} 8+$, NKT cells percentage is lower than the remission group and normal group. Visible asthma acute phase is given priority to with CD4 + lymphocytes. Analysis the reason that most CD4 + cells secrete Th2 cytokines, multiply within the airway eosinophilia, not only in asthma airway inflammatory reaction plays a main role in the process, and can promote the IL - 5 and other support of eosinophils and mature cytokine levels and promotes endothelial cell adhesion factor that eosinophils gathered further. Kim, the study found, such as 18 cases of acute asthma attack, 21 cases of asthma remission and 12 normal study found that children with asthma acute attack of CD4 + cells [(43.75 and 13.5) \%] percentage significantly higher remission group [(37.04 and 7.11) \%] and [ $(33.57$ and 7.54$) \%$ ] normal control group $(\mathrm{P}<0.05)$, percentage of CD8 + cells [] $(21.10$ and $6.10) \%$ was lower than that in group remission [(28.67 and 5.32) \%] and [(28.31 and 9.46) \%] normal control group ( $\mathrm{P}<0.05)$, CD4 / CD8 ratio (2.14 and 0.94) higher than the remission group of soil $(1.33)$ and normal control group $(1.31$ and 0.42$)(\mathrm{P}<0.05)$. Support the results. Das etc to study of adult asthma, acute period of asthma bronchial alveolar bronchial biopsy and sputum induction of Th2 type cytokines level increased obviously, accounts for about $60 \%$ of the total number of T cells count, number of CD $4+$ cells dominate; Rather than in patients with acute onset of NKT cells count is less than $10 \% \sim 15 \%$.

IL - 4 is Th2 cell secretion of cytokines, which can stimulate T cell proliferation, and auxiliary CD4 + cell differentiation, applicability in humeral immunity and immune plays a key role in. IL 10 is mainly composed of Th2 cells and mononuclear macrophages to produce, it can inhibit the production of inflammatory cytokines. Its all in NKT cells contain encoding mRNA, so all can by NKT cells secrete, thus make NKT cells immune regulating cell. This study further amplify different periods of three groups of children of NKT cells factor level analysis found that with the extension of incubation time, three groups of children with serum IL - 4 were significantly increased, and different times of acute onset group level were higher than in remission group and normal group; Serum IL - 10 in cultivating $48 \mathrm{~h}$ rise highest, cultivate $72 \mathrm{~h}$ decline, levels and different periods of acute attack group were higher than in remission group and normal group. Visible children with asthma acute phase there is change on NKT cells function, stimulated 
secretion of inflammatory factor after activation, analyze its possible to promote the body's immune response in inhibition of Th1 response.

Shu-guang Han, such as the study found that children with asthma group after amplification activation of NKT cells secrete IL4 level of the health control group increased significantly (P < $0.01)$, IL - 10 levels decreased significantly $(\mathrm{P}<0.01)$, but the asthma group after amplification activation of NKT cells secrete IFN - 1 level there was no statistically significant difference compared with healthy controls $(\mathrm{P}>0.05)$. Similar to the results, the studies also have, NKT cells involved in the innate immune and acquired immune, is the connection between the bridge and thus can be used as one of the clinical immunotherapy targets.

\section{Conclusion}

In a word, this study shows that the decrease in the number of NKT cells in children with acute attacks of asthma, and its cell function is also change, so we can think that there is a close correlation of asthma and NKT cells, but the mechanism still needs further research.

\section{Acknowledgement}

This research was financially supported by the National Science Foundation.

\section{References}

[1] Jiehua Chen, Yu Deng, Jing Zhao, Zhengxiu Luo, Wansheng Peng,Juan Yang,Luo Ren,Lijia Wang, Zhou Fu,Xiqiang Yang,Enmei Liu. The Polymorphism of IL-17 G-152A was Associated with Childhood Asthma and Bacterial Colonization of the Hypopharynx in Bronchiolitis[J]. Journal of Clinical Immunology. 2012(4)

[2] Yu Li, Ming-Yan Wang, Xin-sheng Fan,Xu Qi, Yan Chen,Huan Zhang,Qing-chun Zhao. Effect of San-ao Decoction, a traditional Chinese prescription, on IL-4 treated normal human bronchial epithelium[J]. Journal of Ethnopharmacology. 2012(1)

[3] Clare M. Lloyd, Catherine M. Hawrylowicz. Regulatory T Cells in Asthma[J]. Immunity. 2013(3)

[4] Yeonseok Chung, Seon Hee Chang, Gustavo J. Martinez, Xuexian O. Yang,Roza Nurieva, Hong Soon Kang,Li Ma, Stephanie S. Watowich, Anton M. Jetten, Qiang Tian, Chen Dong. Critical Regulation of Early Th17 Cell Differentiation by Interleukin-1 Signaling[J]. Immunity. 2013(4)

[5] Wisam Al-Ramli, David Préfontaine, Fazila Chouiali, James G. Martin, Ron Olivenstein, Catherine Lemière, Qutayba Hamid. T H 17-associated cytokines (IL-17A and IL-17F) in severe asthma[J]. The Journal of Allergy and Clinical Immunology. 2012(5)

[6] Bin Chen, Zhirong Zeng, Jiangtao Hou, Minhu Chen, Xiang Gao,Pinjin Hu. Association of interleukin-17F 7488 single nucleotide polymorphism and inflammatory bowel disease in the Chinese population[J]. Scandinavian Journal of Gastroenterology . 2012(6)

[7] Bhanu P. Pappu,Pornpimon Angkasekwinai,Chen Dong. Regulatory mechanisms of helper T cell differentiation: New lessons learned from interleukin 17 family cytokines[J]. Pharmacology and Therapeutics . 2013(3)

[8] Sarah L. Gaffen. An overview of IL-17 function and signaling[J]. Cytokine . 2008 (3)

[9] Kristine Metzger, Marc Frémont, Chris Roelant, Kenny De Meirleir. Lower frequency of IL-17F sequence variant (His161Arg) in chronic fatigue syndrome patients[J]. Biochemical and Biophysical Research Communications. 2012(1)

[10] Tomiyasu Arisawa, Tomomitsu Tahara, Tomoyuki Shibata, Mitsuo Nagasaka, Masakatsu Nakamura, Yoshio Kamiya, Hiroshi Fujita, Masahiko Nakamura, Daisuke Yoshioka, Yuko Arima, Masaaki Okubo, Ichiro Hirata,Hiroshi Nakano. The Influence of Polymorphisms of Interleukin-17A and Interleukin-17F Genes on the Susceptibility to Ulcerative Colitis[J]. Journal of Clinical Immunology. 2012(1) 